\title{
FlexiWall - The design and development of a prototype system that integrates Internet of Things (IoT) technologies, architecture and mobile interaction
}

\author{
Mikael Wiberg \\ Department of Informatics, Umea university, Sweden \\ mwiberg@informatik.umu.se
}

\begin{abstract}
In this paper we present the final step of our Internet of Things (IoT) project called FlexiWall. On an overall level, FlexiWall is a fully working prototype system in the form of an interactive wall element that demonstrates how IoT technologies can be seamlessly integrated in our built environment. In this paper we present the background and the design of the FlexiWall prototype, and we suggest that the FlexiWall prototype demonstrate how Internet of Things (IoT) technologies can be embedded in the everyday architecture of a building as an ambient information display, or as an embedded surface for interaction. Further on, we suggest that FlexiWall works as an illustration of how technologies can be seamlessly embedded in our everyday environments, how it materializes interaction in our built environment, and how the Internet of Things open up new opportunities for systems design the ranges from mobile solutions, to embedded solutions, to interaction across mobile and embedded systems in these new environments. In short and if now directing our attention to the design part of our project as reported in this paper we view the FlexiWall prototype as an interactive, flexible, and wood-based wall element that can bend as to form the light that shines through it as to display different patterns. As such, FlexiWall illustrates a novel ambient display that can be fully embedded in the architecture of a building. We present the background of this design project, including our method that rely on a concept-driven approach to interaction design, and we also present how we draw on theories of light design in architecture in the design of FlexiWall. Further on, we present how our work is related to some existing work in this area, and we present the design and implementation of this interactive wall. Having presented this project we discuss how our work adds to the current body of research within the area of the Internet of Things and mobile interaction with embedded systems before concluding the paper.
\end{abstract}

\section{Introduction}

URI: http://hdl.handle.net/10125/50391

ISBN: 978-0-9981331-1-9

(CC BY-NC-ND 4.0)
The Internet of Things (IoT) comes with the promise that digital technologies can be seamlessly integrated in everyday objects and environments and that such internet enabled objects and environments can enable new forms of computing and interaction. Further on, the IoT movement suggests that computing and Internet technologies can be materialized and as such seamlessly integrated in everyday objects and environments, including our built environment.

In relation to the trend towards integration of IoT technologies in our built environments we can see an increasing interest in ways of conceptualizing and designing for human-environment interaction. One such notion that has recently been highlighted as a promising conceptualization is Human-Building Interaction (HBI) [1]. Human-Building Interaction (HBI) is situated at the intersection of $\mathrm{HCI} /$ interaction design and architecture (see e.g. [2];[11]; [12]) and this notion suggest that as new buildings are increasingly equipped with embedded sensors, actuators, and microcontrollers this development enables these "smart" or IoT-enabled buildings to be interactive, and to support interaction - from automatic doors to far more sophisticated interactive and digital services. Other terms besides Human-Building Interaction include for instance "interactive architecture", "smart environments" or intelligent environment" [5];[12].

No matter how we frame this emerging area we suggest that the current movement towards the Internet of Things (IoT) calls for new approaches and design examples that illustrate how IoT technologies can be seamlessly integrated in our built environment. Our project called "FlexiWall" is one attempt to serve in response to this call.

Further on, and as interactive architecture [5];[12] is increasingly an option when new buildings are planned and built, as well as when old buildings are renovated and restructured to support new needs we also need to think about how we should interact with 
such buildings in terms of interfaces and interaction modalities. Not only does interactive architecture and the area of Human-Building Interaction (HBI) open up for questions concerning security, integrity, and safety, but it also open up a design space concerning how interactivity can be an integrative part of these environments, and accordingly how architectural elements (like walls) can be used as interactive surfaces - not only in meeting rooms, but also while moving around in a building.

In relation to this we see an interesting design space at the intersection of interactivity and spatiality, and at the crossroad of mobility, the Internet of Things, and architecture. In short, the work on FlexiWall as presented in this paper is focused on exploring mobile interaction design in the context of, and in relation to, the intersection of our built environment on the one hand, and the emerging Internet of Things (IoT) on the other hand.

The rest of this paper is structured as follows. First we review and present recent and related work, and in particular we focus on design-oriented research that has focused on Wall-size implementations of interactive systems that supports mobile interaction. We then present our method and approach followed by a section where we describe the design of the FlexiWall prototype. We then describe how a user can interact with FlexiWall, and how it can work as an ambient display. Having presented the design of FlexiWall and its underlying interaction model we iscuss the current implementation and future directions followed by a more general discussion on design of interactive systems for the Internet of Things (IoT) before concluding this paper.

\section{Background}

While some recently conducted research (e.g. [8]) has extensively explored interaction with wall-size displays, our work is more directed towards truly embedded ambient displays and how we can interact with such displays as an integrated part of the architecture of a building. A challenge in this project has accordingly been to think about how a wall as an architectural element can be reimagined to also serve as an interactive surface.

For this particular project we have taken a point of departure in literature in architecture (e.g. [3]) that describe how walls not only serve the purpose of scaffolding the structure of a building, but also how walls consist of solids and voids, supporting both the sheading off as to create space, and allowing for voids, enabling entrances and light to pass through and into the spaces designed ([3]). In particular, we have taken a point of departure in Egan's work [3] on "architectural lightening" and in particular how the use of indirect light could be used, not only for light design of hallways (for a classic example of such use see the work by Anotni Gaudi and how he combined solids and voids in the architecture of his home, Casa Batelló (1906-1925) as to use indirect light to light up hallways (see figure 1 for an illustration).

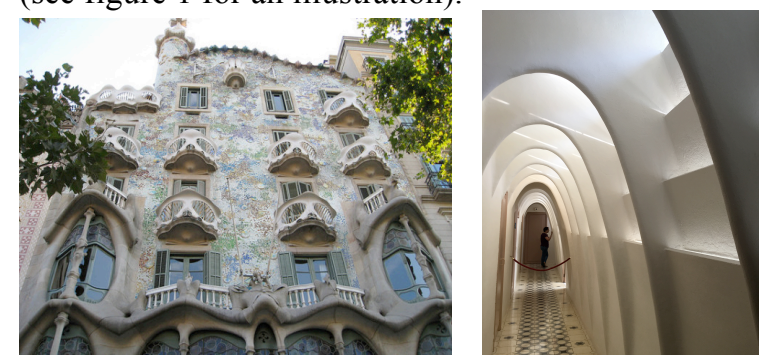

Fig 1. Casa Batelló (left) and the use of voids in the wall to allow for lightening through indirect light (right).

\section{Related work}

The work presented in this paper builds upon, and contributes to some existing literature in the areas of $\mathrm{HCI}$ and interaction design in general, and in more specific terms we contribute to a growing body of research related to prototyping IoT solutions for smart environments. In particular, we build on the existing and growing body of research on Human-Building Interaction (e.g. [1];) and Interactive architecture ([2];[11];[12]). Further on, our research builds on the current, and more general trend towards the Internet of Things, and how such technologies can be integrated in our built environment.

From a conceptual viewpoint our work builds upon the notions of "local mobility" and "Proxemix interaction" [8]. In particular we have been inspired by those concepts in thinking about how people can interact with FlexiWall as part of the architecture of a building.

In terms of existing research prototypes and solutions our work has further on been inspired by the Planks project [10] in terms of the design of a woodbased interactive wall. Further on, we were also inspired by the ABB Powerwall project [4] in terms of how a wall might work as an interactive surface in support of mobile interaction. 
Finally, the design of FlexiWall has also been inspired by theories of light design in architecture. In particular we have been inspired by the work by Egan [3] and his work on concepts in architectural lighting where he describes how light serves as a design material and how architecture is not only about the solids, but also about the design of voids (including not only windows, but all forms of openings that allow for light to interplay with the and light conditions to interplay with the spaces that the architecture enables. In this paper we will accordingly suggest that FlexiWall serves the purpose of demonstrating a solution that builds on these ideas from architecture. As such, it also adds to this strand of existing work by demonstrating an interactive wall that works as an ambient display by letting natural or artificial light shine through the different patterns that the interactive wall produces. How that is enabled is something we will present in section 5 of this paper.

\section{Method}

The design concept behind the FlexiWall prototype was developed through a number of brainstorming sessions with participants from our research group and from industry (including a couple of persons from building construction companies and digital firms) and project meetings. We ran a series of several design meetings, focus group sessions and two workshops as to sketch, generate ideas and test different design ideas. In total 17 persons has been involved in this design project - ranging from programmers and interaction designers, to architects and interior designers in terms of their professions.

Across these sessions of sketching, concept development and prototyping we where inspired by the concept-driven approach to interaction design as proposed by Stolterman \& Wiberg [9] and accordingly we were focused on conceptual development that could both 1) inform the design of FlexiWall, but also support us in 2) designing an interactive prototype that would reflect a particular design concept (rather than following a development cycle all the way from an initial design idea to a finalized product).

Further on, the Concept-driven approach suggested to us that FlexiWall as a concept design could allow for us to explore what one such interactive wall could look like, what it would take to implement it, and how it might work. Accordingly, it could help us gain new knowledge trough the design of this prototype system. As such, our project also rimes well with the growing interest in RtD - Research Through Design in our community.

So, in this particular project it was about exploring the combination of physical design, and architectural concepts for light design from the viewpoint of interactivity, and the outcome from this process was 1) the development of the FlexiWall concept as an interactive, flexible, and wood-based wall element that supports mobile interaction, and 2) the design of FlexiWall as a concept design that serves as a proof-ofconcept in terms of how it might be designed and built.

\section{The design of the FlexiWall prototype}

We have designed, developed and implemented the FlexiWall prototype system as part of a larger ongoing research project that explores digital design and additive manufacturing as an approach to architecture. On an overall level the aim of the project is to explore innovative approaches to architecture and to explore interaction design opportunities in that particular context with a particular focus on Internet of Things (IoT) solutions. Further on, the aim of the project is also to explore ways of embedding and merging digital technology with architectural elements (like walls, doors, ceilings and floors).

For the design of FlexiWall we were interested in exploring wood as an interactive material and how we could design an ambient display the utilized the qualities of wood (for instance that wood can be easily bent) while also serving as a texture in interior design.

We started the project with brainstorming sessions and then moved from that to sketching in 3D. Through that process we were able to review different use scenarios. Further on, we used 3D sketching and modeling as a way of constructing the wall - first as a digital model in the computer, and then to use that 3Dmodel for laser cutting the wooden parts of the wall element (figure 2) and for producing the moving (plastic) parts of the wall by sending those files to a 3D-printer.

FlexiWall is a wall element with a flat wooden surface, and on the inside of the wall element there is a computer-controlled rig with a moving wheel (see fig 2). The current implementation of FlexiWall has one wheel and a one-axis design, but it can easily be extended to a two-axis implementation. 


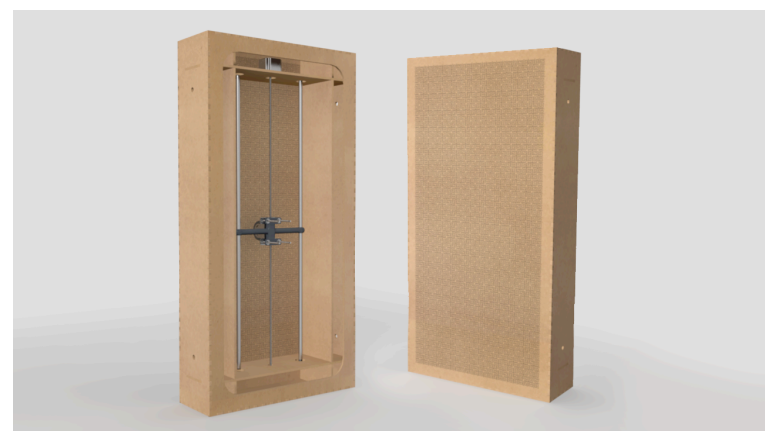

Figure 2. 3D model illustrating the implementation the FlexiWall prototype

Further on, the wooden surface of FlexiWall has a laser cut pattern (see fig 3, left) that makes the wood easy to bend, and it allows for light behind this wooden surface to shine through the material.

In moving forward, figure 3 (left) illustrate the 3D model of a wheel that pushes the wooden surface of FlexiWall outwards as to bend it and accordingly create "wave" patterns across the wood, both physically as the wood bends, but also as it changes how light from the backside of the wall element shines through the wood in different ways. In figure 3, right, we illustrate what the 3D model of that wheel looks like when we had 3D printed and assembled the parts for the FlexiWall.
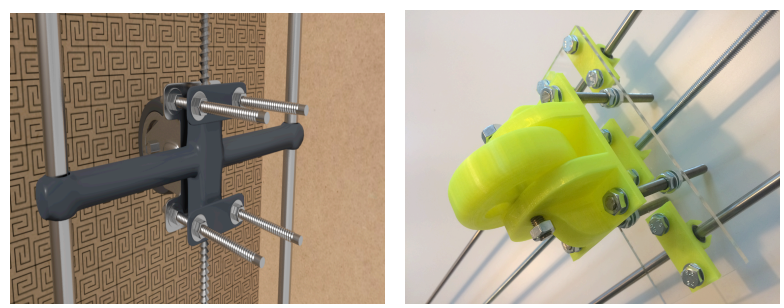

Figure 3. From 3D model to implementation

We attached this wheel to the rig as illustrated in fig 3 and 4 where two strings, to the left and to the right of the wheel keeps it in position, and where the middle string where attached to a servo motor. With this configuration we could control the rotation of the middle string via an arduino board and through that solution move the wheel up and down on the backside of the wooden surface of FlexiWall.
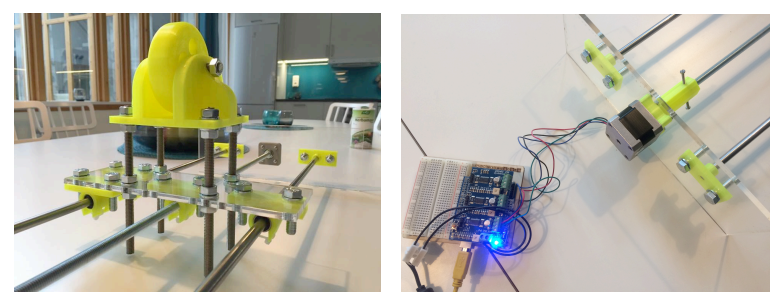

Figure 4. The internal rig, wheel, the servo motor and the Arduino board to control the movement of the wheel.

We used a standard protocol typically used for sending commands to $3 \mathrm{D}$ printers for controlling the servomotor, and accordingly this configuration allowed for moving the wheel up and down the rig to a particular position. While that might not be important in order to create a wave shape as the wheel bent the wood as it moved across the wooden surface we thought that this implementation also allowed for some scaling, enabling easy scaling of this project to multiple axis, multiple wheels, and even to connect several FlexiWall elements into large walls where the different positions on each element when taken together could form a large-scale pattern or waveeffect across several FlexiWall elements.

\section{Interaction with FlexiWall}

The current implementation of the FlexiWall prototype supports mobile interaction with the wall via a simple web-based interface. The interface allows a user to send commands to the display, and it provides support for controlling the arduino board inside of FlexiWall. This setup allows for simple interaction with the display. For instance, with this interface the wall element can bring up an alert, the user can respond to the alert, and in return FlexiWall can change state/light pattern as to provide feedback to the user. Further on, and since we have implemented this in the form of a web-based interface, which also means that a user can interact with this wall via any type of mobile device (including smartphones, tablets, or just an ordinary laptop).

From an interaction design perspective on this project we envision use scenarios where people move seamlessly between mobile and wearable technologies that can be held or worn, with this wall-based interactive surface. Further on, we think about mobile devices as lightweight, mobile and high-resolution devices suitable for information processing and editing, whereas an ambient display like FlexiWall is more stable, low-res, and supports slow-changing information signs (e.g. directions, or alerts about new information), that again can be further supported with mobile or wearable technologies.

For future iterations of the design we have also considered a range of additional interaction modalities including how movement tracking and gestures might be suitable modes for interacting with FlexiWall. Further on, and when it comes to the different services 
that we see that FlexiWall can support we have so forth looked mainly at basic services suitable for this type of ambient display, including its ability to provide information in the form of notifications and alerts, basic information about direction (for instance in case of fire), or other forms of basic information that suits this kind of ambient display (for instance indoor temperature, or basic weather information). However, what FlexiWall can support is at the current moment dependent on its current implementation.

\section{FlexiWall as an ambient display - Current prototype implementation and future directions}

In the current implementation FlexiWall supports one-axis movement of the wheel that changes how the light shines through the wooden surface. That is a simple design, but yet efficient for showing information that can be presented along a scale (for instance temperature, stock changes, time, etc.). However, for more complex information additional wheels and axis might be needed.

Further on, the current implementation allows for either natural or artificial light to shine through the patterns that the wheel creates as it moves across the wooden board, but in order to support more complex information to be communicated via this ambient display it would be interesting to also explore the combination of different lights (natural light, artificial light, and interactive light) in combination with explorations of different light conditions (daylight, dawn, sunrise/sunset, and indoor/outdoor light conditions).

Finally, the current implementation is limited to "static" light (either natural light or artificial light) shining through the wood, but it would be interesting to also look into opportunities for playing with the temporality of the light (dimming it or switching it on/off) as to support more complex, and a wider array of possible states for this ambient display (for instance it enabling wave-like light patterns, in combination to slow or fast "wood waves" across the surface of this wall element.

\section{Discussion}

The "Internet of Things" suggests that everyday objects can be equipped with computational capabilities, and accordingly how that enables such objects to be connected and part of digital services. In a similar way, we suggest that traditional architectural elements - such as a wall - can be re-imagined in a computational moment. In this paper we have illustrated this in the form of a wall element with computational, and interactive, capabilities.

In terms of future work there are many options in terms of moving forward with the next design iteration of FlexiWall. For instance, we are interested in exploring how to design for more complex light/bend patterns, how to design for a combination of natural, artificial and interactive lightening, to explore more advanced interaction modalities, and design for a broader set of digital services that can run across this interactive wall and a multitude of mobile devices.

At the same time we have in a similar manner as [7] noticed how more minimalistic design allow for a more in-depth exploration of particular research questions. For instance, to explore questions related to the materiality of particular material instantiations of digital designs. This lesson learned from our previous project also rimes well with the conclusion drawn by [13] in their paper "what makes a prototype novel" where they suggest that a minimalistic design both highlight the unique aspects of a particular design while also allowing for a more in-depth exploration of a particular design concept. However, and while that is good for an initial exploration of a design concept the next step in this project is aimed at a user study as to collect data about how this interactive wall can support local indoor mobile interaction and also to study how users interact with this wall, aspects related to user experience and how the wall might support group collaborations, coordination, alerts and distribution of information in the form of an ambient display.

\section{Conclusions}

In this paper we have presented the design of FlexiWall as an interactive wall element that supports mobile interaction. . On an overall level we have presented FlexiWall as a prototype system in the form of an interactive wall element that demonstrate how IoT technologies can be seamlessly integrated in our built environment. In this paper we have presented the background and the design of the FlexiWall prototype, and we have suggested that the FlexiWall prototype demonstrates how Internet of Things (IoT) technologies can be embedded in the everyday architecture of a building as an ambient information display, or as an embedded surface for interaction. Further on, we have suggested that FlexiWall works as an illustration of how technologies can be seamlessly embedded in our everyday environments, how it 
materializes interaction in our built environment, and how the Internet of Things open up new opportunities for systems design the ranges from mobile solutions, to embedded solutions, to interaction across mobile and embedded systems in these new environments.

From a conceptual viewpoint we suggest that FlexiWall works as a demonstration of an interactive, flexible, and wood-based wall element that can bend as to form the light that shines through it into simple light patterns or messages. In this paper we suggest that FlexiWall contributes to the established body of work on interactive walls and in particular interactive and ambient displays by illustrating a novel ambient display that can be fully embedded in the form of an architectural element in a building.

In this paper we have present the background of this project, including our concept-driven approach behind this project, and how we have drawn on theories of light design in architecture as to inform this project. We have also pointed to the next step in this project, including an empirical evaluation of this prototype.

With the presentation of this design project we suggest that we contribute to the further development of our field, both in terms of the development of the FlexiWall prototype, but also in terms of the further elaboration of concepts for the design of interactive walls and ambient displays that support mobile interaction. Further on, and on a more general level we think that this presentation of our prototype design and the presentation of the development behind this project can contribute to practice in terms of working as an example that illustrate how IoT technologies might be materialized in architectural spaces, how this enable the development of new interactive environments, and how this open up new opportunities for systems development at the intersection of IoT, mobile/wearable technologies and architecture in the development of new environments.

\section{Acknowledgements}

The research reported in this paper has been partly funded by the +Project, WP: Lighthouse. I would like to thank Andreas Lund, Johan Bodén and Fatemeh Moradi for our collaboration throughout this project and for our collaboration in the design and development of the FlexiWall prototype system. In particular I would like to thank Andreas Lund for the work on the electronics, Johan Bodén for the work on the 3D-printed parts, and the whole project group for the ideation and design process, and for our creative collaboration that led to the development of the prototype system as presented in this paper.

\section{References}

1. Alavi, H, Churchill, E., Kirk, D., Nembrini, J., Lalanne, D. (2016 Deconstructing Human-Building Interaction, ACM Interactions, Nov-Dec 16.

2. Dalton, S, Schnädelbach, H., Wiberg, M. et al (eds)

(2016) Interaction \& Architecture: human-computer interaction in time and place, Springer verlag.

3. Egan, D. (2001) Architectural Lighting, McGrawHill Science.

4. Fallman, D., Kruzeniski, M., \& Andersson, M. (2005) Designing for a Collaborative Industrial Environment: The Case of the ABB Powerwall, Proceedings of DUX 2005, Conference on Designing for User Experience, San Francisco, CA, November 3-5: ACM Press.

5. Fox, M. and Kemp, M. (2009) Interactive architecture, Princeton Architectural Press.

6. Lischke, L., Grüninger, J., Klouche, K., Schmidt, A., Slusallek, P., Jacucci, G. (2015) Interaction Techniques for Wall-Sized Screens, In Proceedings of ITS '15 - the 2015 International Conference on Interactive Tabletops \& Surfaces.

7. Lund, A. \& Wiberg, M. (2004) Ambient Displays Beyond Conventions, Paper presented at the "Designing for attention" workshop at HCI 2004, The 18th British HCI Group Annual Conference, Leeds Metropolitan University, UK 6-10 September 2004.

8. Marquardt, N., and Greenberg, S. (2010) Proxemic interaction: designing for a proximity and orientation-aware environment, In Proceedings of ITS '10 ACM International Conference on Interactive Tabletops and Surfaces, Pages 121-130.

9. Stolterman, E. \& Wiberg, M (2010) Conceptdriven Interaction Design Research, Human Computer Interaction (HCI), Vol 25, Issue 2, p. 95-118.

10. Vallgårda, A. (2008) PLANKS: A Computational Composite, NordiCHI'08, Lund, Sweden, October 20 - 22, 2008

11. Wiberg, M. (2015) Interaction Design Meets Architectural Thinking, ACM interactions, March + April 2015, p. 60-63.

12. Wiberg, M. (2011) Interactive Textures for Architecture and Landscaping - Digital Elements and Technologies, Information Science Reference, IGI-global, USA.

13. Wiberg, M. and Stolterman, S. (2014) What makes a prototype novel? A knowledge contribution concern for interaction design research, In proceedings of NordiCHI' 14. 